\title{
Rôle d'une levure dans un accident de fabrication de yoghourt brassé
}

\author{
par \\ G. Dubois, France Desaulniers-Therrien \\ et R. CharbonneaU*
}

\section{INTRODUCTION}

A la suite d'accidents de fabrication survenus dans une laiterie de la région de Montréal, sur un yoghourt brassé, nous avons été amenés à effectuer une analyse microbiologique du contaminant afin de déterminer la nature de cet agent et de pouvoir y remédier. Les symptômes étaient les suivants : gonflement excessif de l'emballage et odeur désagréable.

\section{MATERIEL ET METHODES}

L'analyse microbiologique a consisté en un examen microscopique à l'état frais et par la coloration de Gram, en une numération de la flore totale sur le milieu PCA (APHA [2]), en celle des coliformes par la méthode au bouillon lactosé bilié au vert brillant (Thatcher et Clark [9]), en celle des streptocoques fécaux sur milieu de Kenner et al. [4] confirmé par culture sur milieu de Litsky [5] et en celle de la flore fongique (levures et moisissures) sur milieu de Sabouraud (FDA [3]).

Les Salmonella (Mc Coy [7]) et les staphylocoques pathogènes (Thatcher et Clark [9]) ont été également recherchés. La levure isolée a été étudiée et déterminée selon les techniques décrites par Lodder [6].

\section{RESULTATS ET DISCUSSION}

Des examens par la coloration de Gram et à l'état frais ont permis la mise en évidence de bactéries Gram + dont la présence est normale (lactobacilles et streptocoques) et de levures en grandes quantités.

\footnotetext{
* Laboratoire de microbiologie, Centre de Recherches en Sciences Appliquées à l'Alimentation (CRESALA ${ }^{r}$ ), Université du Québec à Montréal, C.P. 8888, Montréal, P. Québec, H2V 1L6 (Canada).
} 


\section{TABLEAU 1}

Analyse bactériologique du yoghourt

\begin{tabular}{l|c}
\hline \multicolumn{1}{c|}{ Tests } & Nombre de cellules par $\mathrm{g}$ \\
\cline { 2 - 2 } & \\
\cline { 2 - 2 } Flore totale & $3 \times 10^{7}$ \\
Coliformes & 10 \\
Streptocoques fécaux & 10 \\
Staphylocoques & négatif \\
Salmonella & négatif \\
Levures & $7 \times 10^{6}$ \\
Moisissures & négatif \\
& \\
\hline
\end{tabular}

TABLEAU 2

Substrats fermentés par Kluyveromyces bulgaricus (Santa Maria) Van Der Walt

\begin{tabular}{l|c}
\hline \multicolumn{1}{c|}{ Substrats } & Réaction par rapport au glucose \\
\hline Glucose & intense et rapide \\
Galactose & intense et rapide \\
intense et rapide \\
Sucrose & intense et rapide \\
Raffinose & intense et rapide \\
Inuline & lent \\
Lactose & négatif \\
Maltose & négatif \\
Cellobiose & négatif \\
Trehalose & négatif \\
Melibiose & négatif \\
Melezitose & négatif \\
$\alpha$-Methyl-D-glucoside & négatif \\
Amidon soluble & \\
& \\
\hline
\end{tabular}

Le tableau 1 représente les résultats de l'analyse. La flore totale est légèrement inférieure à celle retrouvée habituellement dans les yoghourts (Accolas et al. [1]). Le taux de coliformes et de streptocoques fécaux est très bas. Il n'a pas été trouvé de Salmonella et de staphylocoques entérotoxiques. L'accident observé est essentiellement causé par la très forte flore de levures présente dans le yoghourt.

Une vingtaine de colonies de levures ont été prélevées et identifiées ; toutes se sont révélées être des : Kluyveromyces bulgaricus (Santa Maria) Van Der Walt. Cette espèce a été déjà isolée du yoghourt par Santa Maria Ledochowski [8]. Les analyses microscopi- 
TABLEAU 3

Substrats assimilés par Kluyveromyces bulgaricus (Santa Maria) Van der Walt

\begin{tabular}{|c|c|}
\hline Composés & Assimilation par rapport au glucose \\
\hline $\begin{array}{l}\text { Glucose } \\
\text { Galactose } \\
\text { Sucrose } \\
\text { Lactose } \\
\text { Raffinose } \\
\text { Inuline } \\
\text { D-Xylose } \\
\text { Ethanol } \\
\text { Glycerol } \\
\text { Ribitol } \\
\text { D-glucitol } \\
\text { DL-Acide lactique } \\
\text { Acide succinique } \\
\text { Ethylamine hydrochloride } \\
\text { Trehalose } \\
\text { L.-Arabinose } \\
\text { D-Mannitol } \\
\text { L. Sorbose } \\
\text { Maltose } \\
\text { Cellobiose } \\
\text { Melibiose } \\
\text { Melezitose } \\
\text { Amidon soluble } \\
\text { D-Arabinose } \\
\text { D-Ribose } \\
\text { L-Rhamnose } \\
\text { Erythritol } \\
\text { Galactitol } \\
\alpha \text {-Methyl-D-glucoside } \\
\text { Salicine } \\
\text { Acide citrique } \\
\text { Inositol } \\
\text { Nitrate de potassium }\end{array}$ & $\begin{array}{l}\text { rapide } \\
\text { rapide } \\
\text { rapide } \\
\text { rapide } \\
\text { rapide } \\
\text { rapide } \\
\text { lente } \\
\text { rapide } \\
\text { rapide } \\
\text { lente } \\
\text { rapide } \\
\text { rapide } \\
\text { rapide } \\
\text { rapide } \\
\text { rapide } \\
\text { lente } \\
\text { faible } \\
\text { négatif } \\
\text { négatif } \\
\text { négatif } \\
\text { négatif } \\
\text { négatif } \\
\text { négatif } \\
\text { négatif } \\
\text { négatif } \\
\text { négatif } \\
\text { négatif } \\
\text { négatif } \\
\text { négatif } \\
\text { faible } \\
\text { négatif } \\
\text { négatif } \\
\text { négatif }\end{array}$ \\
\hline
\end{tabular}

ques ont montré la présence d'ascospores sphéroïdaux (entre 1 et 4) contenant, pour certains, des globules de lipides. Les souches ont fermenté plusieurs sucres comme le lactose et n'ont pas assimilé le cellobiose. Les tableaux 2 et 3 résument les analyses qui ont permis l'identification. Ceci concorde avec la nature de l'accident mis en cause.

Un examen de la chaîne de fabrication du yoghourt a permis de trouver les points où s'effectuait la contamination. Les accidents ont été éliminés à la suite du nettoyage. 


\section{Ré s u m é}

Les accidents dans cette fabrication de yoghourt (gonflement de l'emballage et odeur désagréable) sont dus à une très forte contamination par la levure Kluyveromyces bulgaricus (Santa Maria) Van Der Walt.

\section{S u $\mathbf{m} \mathbf{m}$ a $r$ y}

\section{YEAST CONTAMINATION OF YOGHURTS: GAZ BULGING AND BAD TASTE}

Making flaws (gaz bulging and bad taste) observed of these productions yoghourt depend on yeast contamination by Kluyveromyces bulgaricus (Santa Maria) Van Der Walt.

\section{Remerciements}

Nous tenons à remercier Marie Alix d'Halewyn des laboratoires du Ministère des Affaires sociales du Québec qui nous a aidés lors de ce travail.

Reçu pour publication en mars 1980.

\section{Bibliographie}

[1] Accolas (J. P.), Veaux (M.), Delmas (C.), Sansoulet (O.), Grappin (R.) et Petransxiene (D.) (1978). - Comptage de Streptococcus thermophilus et de Lactobacillus bulgaricus dans les yoghourts. C.R. du $20^{\mathrm{e}}$ Congr. Int de Laiterie, Paris, p. 354.

[2] A.P.H.A. (1979), - Standard methods for the examination of dairy products. 14th ed W. J. Hausler Jr, editor. American Public Health Association, Washington, D.C.

[3] F.D.A. (1976). - The Bacteriological analytical manual for foods. Foods and Drug Administration, 4th ed. A.O.A.C. Publ., Washington (U.S.A.).

[4] Kenner (B. A.), Clark (H. F.) and Kabler (P. W.) (1961). - Fecal streptococci. I. Cultivation and enumeration of streptococci in surface water. Appl. Microbiol., 9, 15.

[5] Litsky (W.), Mallmann (W.) and Fifield (C.) (1955), - Comparison of the most probable numbers of $E$. coli and enterococci in river waters. Ann. J. Publ. H.L.T.H., 45, 1049.

[6] Lodder (J.) (1970). - The Yeast. North Holland Publ. Co., Amsterdam.

[7] Mc CoY (J. H.) (1962). - The isolation of Salmonella. J. Appl. Bacteriol., $25,213$.

[8] Santa Maria Ledochowski (J.) (1956). - Saccharomyces fragilis, Jörgensen. var. bulgaricus, nov. var. Ann. Inst. Nac. Invest. Agr., 5, (2), 163.

[9] Thatcher (F. S.) and Clark (D. S.) (1978). - Microorganisms in Foods. University of Toronto Press, Toronto (Canada). 